\title{
Dual-Resonator MEMS Magnetometer Based on Self-clocking Sigma-delta Modulation
}

\author{
Fang Chen, Wei Zhou, Hongshuo Zou, Michael Kraft, and Xinxin Li
}

\begin{abstract}
This paper presents the design and experimental evaluation of a micromachined Lorentz force magnetometer with a dual-resonator structure, interfaced with an electromechanical sigma-delta modulation (EM- $\Sigma \Delta \mathrm{M})$ force rebalancing control loop. The magnetometer chip comprises a matched pair of resonators on the same silicon die. The closed-loop control circuit relies on a self-oscillating architecture; the frequency of which is determined by first resonator. It is used to generate a drive current injected to the second resonator, and also generates all internal circuit clocks based on a self-clocking scheme. System level simulation indicate a signal-to-noise ratio (SNR) larger than $100 \mathrm{~dB}$ in a bandwidth of $64 \mathrm{~Hz}$ with a $10 \mathrm{mT}, 32 \mathrm{~Hz}$ magnetic field input $(B)$. The system was implemented in hardware based on a dual quantization technique, which has the advantage of reduced quantization error of multi-bit quantization in a single sigma-delta modulator. Experimental results demonstrate that proposed dual-resonator magnetometer achieved a 7-fold improvement in bias instability (increasing the averaging time to reach the bias instability from $1 \mathrm{~s}$ to $8 \mathrm{~s}$ ) and the bandwidth (BW) is increased to $\sim 80 \mathrm{~Hz}, 16$-fold higher compared to an open-loop magnetometer with a single resonator. With a $1 \mathrm{~mA}$ bias current, the output noise is less than $1 \mu \mathrm{T} / \sqrt{ } \mathrm{Hz}$ for the z-axis magnetic field.
\end{abstract}

Index Terms - Lorentz force, dual-resonator, magnetometer, EM- $\Sigma \Delta M$, force rebalance, self-clocking.

\section{INTRODUCTION}

$\mathrm{M}$ ICRO-ELECTRO-MECHANICAL systems (MEMS) Lorentz force magnetometers belong to inertial sensors, which have recently attracted considerable interests in many applications, such as personal navigation, mineral detection, and aerospace [1]. Compared to traditional magneto-resistive (MR), fluxgate and Hall effect sensors, MEMS Lorentz force magnetometers have the advantages that they are free of magnetic hysteresis and can be co-fabricated with other MEMS inertial sensors (Coriolis force gyroscopes and accelerometers) on the same silicon die [2]. Considerable effort have been made to improve the sensitivity and resolution of Lorentz force

This work was supported by the National Natural Science Foundation of China under grant 61504159 and 61604165 , in part by Natural Science Foundation of Shanghai under grant 19ZR1467300, in part by MOST of China under grant 2016YFA0200800.

F. Chen, W. Zhou, H.S. Zou and X.X. Li are with the State Key Laboratory of Transducer Technology, Shanghai Institute of Microsystem and Information Technology, Chinese Academy of Sciences, Shanghai 200050, China (e-mail: fangchen@mail.sim.ac.cn; xxli@mail.sim.ac.cn).

M. Kraft is with the MICAS group, Catholic University of Leuven, Belgium (e-mail: m.kraft@kuleuven.be) magnetometers by the maximizing the quality factor value $(Q)$. In amplitude modulation $(A M)$ Lorentz force magnetometers, the external magnetic field $(B)$ together with applied electrical currents create a proportional change in the resonator's output amplitude. It has been demonstrated that open-loop AM magnetometers have already achieved high sensitivity and a noise-equivalent magnetic field of below $30 \mathrm{nT} / \sqrt{ } \mathrm{Hz}$, which is almost 10 times better than that of existing Hall-effect sensors [3]. The force-rebalanced, closed-loop magnetometer with AM readout driven by both electrostatic force and Lorentz force has been demonstrated, reducing the sensitivity of the sensor with temperature [4-5]. However, large output offset and significant temperature sensitivity due to silicon temperature coefficient of frequency $(T C F)$ degrading bias stability, remain problematic. In addition, the bandwidth is still limited by the micromachined architecture. In recent published work, open- and closed-loop Lorentz force magnetometers operating off-resonance were proposed [6-8]. This off-resonance mode of operation enables maximum sensing bandwidth and but reduces sensor resolution. Therefore, the magnetometer performance is still not high even with a large frequency split.

Frequency modulation $(F M)$ readout magnetometers can be realized either by using the Lorentz force to change its resonant frequency [9-10], or by applying a quadrature Lorentz force to an electrostatically excited MEMS oscillator [11-12] which is similar to quadrature frequency modulation $(Q F M)$ gyroscopes (having quadrature Coriolis force as the external force). $F M$ readout magnetometers potentially have some advantages over $A M$ readout magnetometers, such as there is no trade-off between bandwidth and quality factor $(Q)$, and large dynamic range. However, their sensitivity is still low and bias instability is still influenced by the resonator's $T C F$.

We have recently reported on the research results of a new dual resonator MEMS magnetometer. This paper expands on preliminary research presented in [13] by providing additional analysis and results. In this work, a self-clocked magnetometer composed of two identical resonators on the same silicon die; the two resonators are controlled by a digital self-oscillation loop and a force rebalanced loop, respectively. The oscillation loop signal is used as the reference clock and to generate the Lorentz excitation current. This self-clocking scheme provides a fixed ratio between the system sampling frequency and the resonant frequency (Lorentz signal carrier), which suppresses the temperature-induced offset drift. A new advanced SOI wafer, dicing free, dry release process was developed in our laboratory 
to allow the magnetometer chips to be separated from each other. Furthermore, combined with the advanced circuit techniques and dual quantization high-order EM- $\Sigma \Delta \mathrm{M}$ scheme [14], makes Lorentz force magnetometer suitable for high-end applications, enabling magnetic field measurements that is insensitive to external temperature fluctuations, vibration interfere, etc. The magnetometer presented hereafter can be co-fabricated with other inertial sensors, thus enabling a unified EM- $\Sigma \Delta \mathrm{M}$ control scheme [15-17].

The paper is organized as follows: Section II describes the structure design and fabrication process. Section III describes the system level modelling and the analytical investigation. Section IV presents the hardware implementation of the control system and experimental results. Section VI draws some final conclusions.

\section{DEVICE DESCRIPTION AND FABRICATION}

\section{A. Device and system dynamics}

The proposed schematic of dual-resonator structure, shown in Fig.1, consists of two identical three-port resonators, each resonator consists of an $\mathrm{H}$-shaped moving structure suspended by several folded springs. Parallel plate driving and sensing capacitive electrodes in the first resonator, labeled as $D R$ and $S$, are used to generate the electrostatic force driving one resonator into an in-plane oscillation. Further parallel plate capacitive electrodes in the second resonator, labeled as $S$ and $F B$, are used for magnetic field $(B)$ sensing and generating the electrostatic feedback force to counterbalancing the generated Lorentz force, respectively.

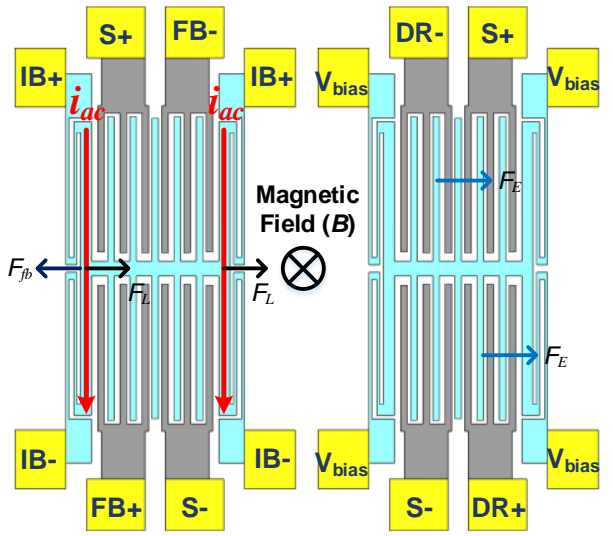

Fig.1. Schematic of dual-resonator Lorentz force magnetometer. AC current is injected at pads IB+ and IB-.

During operation, the first resonator is driven by electrostatic force $\left(F_{E}\right)$ as a closed-loop oscillator, so that structure vibrates continuous at its resonant frequency, providing a reference frequency for the driving current $\left(i_{a c}\right)$. The driving current $\left(i_{a c}\right)$ injected at contacts $I B+$ and $I B$-, interacts with the external magnetic field rate $(B)$ to produce an amplitude modulated Lorentz force $\left(F_{L}=B \bullet i_{a c} \bullet L_{e}\right)$, where $L_{e}$ is the effective length of the driving current $\left(i_{a c}\right)$ path through the resonator structure. The generated Lorentz force $F_{L}$ will be counterbalanced by the electrostatic feedback force $F_{f b}$; the magnetic field strength $B$ can be inferred from the change in the feedback force amplitude
$\left(V_{f b}\right)$. The system dynamic and the steady state oscillation amplitude of the first resonator can be written as:

$$
\begin{gathered}
m^{\prime} \frac{d^{2} x^{\prime}(t)}{d t^{2}}+b^{\prime} \frac{d x^{\prime}(t)}{d t}+k^{\prime} x^{\prime}(t)=F_{E} \\
x^{\prime}(t)=\frac{F_{E} Q}{k}
\end{gathered}
$$

The second resonator's response to the electrostatic feedback force and Lorentz force is modeled by the following differential equation

$$
\begin{gathered}
m \frac{d^{2} x(t)}{d t^{2}}+b \frac{d x(t)}{d t}+k x(t)=F_{f b}-F_{L}-F_{e x} \\
F_{f b}=F_{L}-F_{e x}=B \times i_{a c} \times L_{e}-F_{e x}
\end{gathered}
$$

where $m, b$ and $k$ is the effective mass, the damping factor and stiffness of the resonator; $F_{e x}$ represents other external forces (e.g. caused by unwanted vibrations). The feedback force is generated and limited by the maximum amount of feedback voltage generated by the control electronics. In order to have zero displacement in the Lorentz force sense direction, the controller tries to enforce the following conditions:

$$
\begin{gathered}
x(t)=0 \\
F_{f b}=V_{\text {Bias }} \frac{d C_{x}}{d x} V_{f b}=F_{L}-F_{e x}
\end{gathered}
$$

where $V_{f b}$ is the feedback voltage to generate $F_{f b}$. The output voltage $V_{o}$ in the force rebalance loop can be derived according to (5) and (6), here only considering the Lorentz force:

$$
V_{o}=\left(B \times i_{a c} \times L_{e}\right) /\left(V_{\text {Bias }} \frac{d C_{x}}{d x}\right)
$$

The expression of scale factor $(S F)$ can be expressed by:

$$
S F=\left(i_{a c} \times L_{e}\right) /\left(V_{\text {Bias }} \frac{d C_{x}}{d x}\right)
$$

Equation (8) indicates that the closed-loop scale factor $(S F)$ does not depend on the resonator characteristics (frequency, quality factor), but still depends on the effective length $L_{e}$, drive current amplitude $i_{a c}$, bias voltage $V_{\text {Bias }}$, and the capacitance used to generate the electrostatic force. In addition, the $\Sigma \Delta \mathrm{M}$ 1-bit bitstream feedback is achieved by two alternating voltages, which are applied to the non-collocated parallel feedback electrodes $(F B+$ and $F B$-) to generate digital feedback force, the feedback gain is given as

$$
K_{f b}=\operatorname{sgn}\left(D_{\text {out }}\right) \frac{\varepsilon_{0} A_{f b}(\Delta V)^{2}}{2\left(d_{0}+\operatorname{sgn}\left(D_{\text {out }}\right)(x)\right)^{2}}
$$

where $\varepsilon_{0}$ is the dielectric constant of vacuum, $A_{f b}$ is the area of the feedback electrode, $\Delta V$ is the voltage difference between $V_{r e f}$ and $V_{\text {Bias }}, d_{0}$ is the nominal gap, and $D_{\text {out }}$ is the momentary value of the pulse density modulated $(P D M)$ digital bit-stream, which is either a positive or negative reference voltage $\left( \pm V_{\text {ref }}\right)$. In this case, digital code of scale factor $S F_{\text {digital }}$ expression can be modified as:

$$
S F_{\text {digital }}=\left(i_{a c} \times L_{e}\right) / K_{f b}
$$

Based on the above analyses, the stability of the scale factor is only related to the stability of the drive current amplitude, feedback voltage, and $d C_{x} / d x$, which are prone to changes with temperature, stress, noise, etc. The other parameters, such as the 
effective length $L_{e}$ can be considered as robust and thus are insensitive to environmental disturbances.

\section{B. Fabrication and frequency response}

The fabrication of prototypes was done using an advanced in-house silicon-on-insulator-wafer-based process, which also can be used for fabricating accelerometers and gyroscopes in the same silicon die. The SOI wafer used in this work consists of a structural layer of $30 \mu \mathrm{m}$, a silicon handle wafer of $400 \mu \mathrm{m}$, and an intermediate insulating and sacrificial BOX layer of $2 \mu \mathrm{m}$. Fig.2 illustrates a schematic diagram of the fabrication process flow. The process starts with the preparation of a SOI wafer by highly boron-doped (p-type) with resistivity of $5 \mathrm{~m} \Omega \cdot \mathrm{cm}$ is used to reduce the resistance of the current-carrying flexure. Both sides of the SOI wafer have to be consecutively pattered and etched by Deep Reactive Ion Etching (DRIE) to form the device structure layer and etch away a block of the handle wafer underneath the structure. The DRIE was chosen over other types of etching techniques like isotropic or anisotropic wet etching due to the vertical sidewalls in device features. The back side of the SOI wafer have to be consecutively patterned and etched by DRIE to etch away a block of the handle wafer underneath the vibrating structure. After DRIE, the micro-structure can be released by etching the sacrificial silicon dioxide in HF vapour. The SEM image of the fabricated magnetometer MEMS chip is shown in Fig.3. The dimensions of the magnetometer consist of two identical $1.4 \mathrm{~mm} \times 0.7 \mathrm{~mm}$ silicon resonators integrated on the same silicon die. The package-level technology for the robust vacuum sealing of the magnetometers was implemented to allow experimental characterization of stand-along sensors. The packaging procedure comprises the eutectic attachment of a MEMS die to a ceramic package, wire-bonding, and eutectic sealing of the device in a vacuum preceded by activation of the getter deposited on the lid. Table I shows the measured values of the magnetometer parameters. (a)

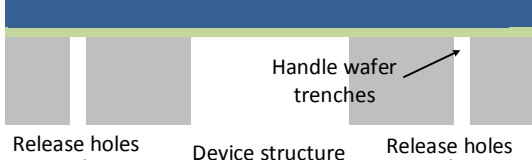

(b)

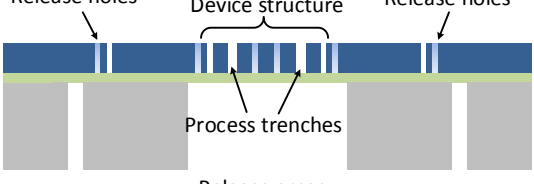

(c)

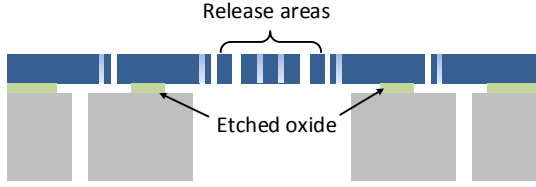

(d)

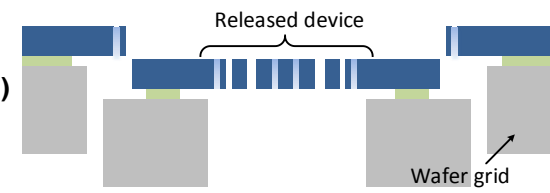

Fig.2. Cross-section of the fabrication flow steps using a SOI wafer for the dual resonator capacitive magnetometer: (a) handle wafer etching in DRIE. (b) Structural layer etching in DRIE to pattern the device features. (c) The release areas released in consecutive order in HF VPE. (d) Device separation after HF VPE release.

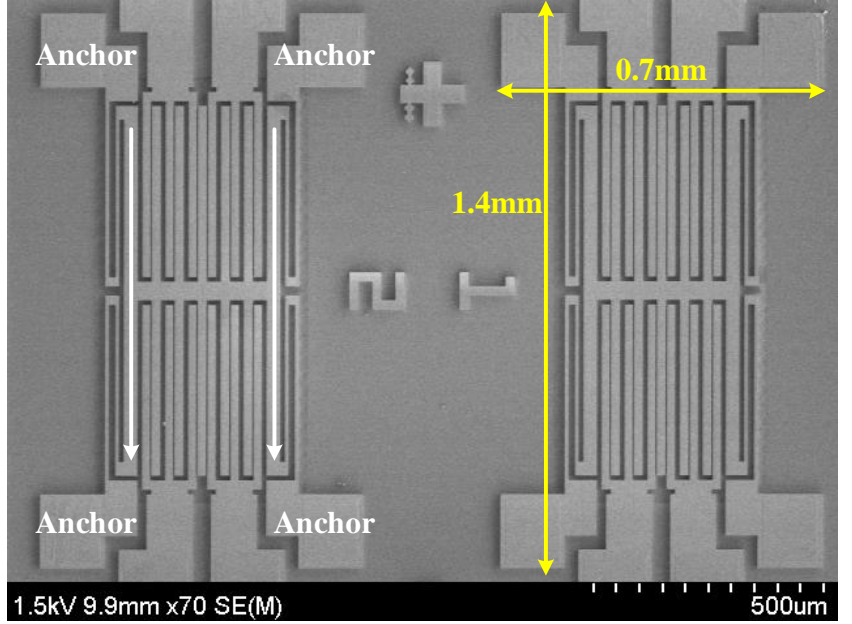

Fig.3. Top SEM image of the fabricated silicon dual-resonator magnetometer with a chip size of $2 \mathrm{~mm} \times 2 \mathrm{~mm}$. white arrows indicate the current through the flexure.

Table I: Main parameters of the dual-resonator Lorentz force magnetometer

\begin{tabular}{ccc}
\hline \hline Parameter & Resonator 1 & Resonator 2 \\
\hline Resonant frequency & $19860 \mathrm{~Hz}$ & $19961 \mathrm{~Hz}$ \\
Quality factor & $\sim 2000$ & $\sim 2000$ \\
Effective length & $800 \mu \mathrm{m}$ & $800 \mu \mathrm{m}$ \\
Capacitance (in-plane) & $160 \mathrm{fF}$ & $160 \mathrm{fF}$ \\
Capacitive gap & $2 \mu \mathrm{m}$ & $2 \mu \mathrm{m}$ \\
Device thickness & $30 \mu \mathrm{m}$ & $30 \mu \mathrm{m}$ \\
Device area & $\sim 1 \mathrm{~mm}^{2}$ & $\sim 1 \mathrm{~mm}^{2}$ \\
\hline \hline
\end{tabular}

The magnetometer frequency response is characterized with an open loop interface configuration by sweeping the frequency of a constant amplitude signal and measuring the corresponding output amplitude. Although these two resonators are designed to matching and are integrated on a same die, micromachining process imperfections are inevitable and result in a difference between these two resonators. Measured frequency responses are shown in Fig.4, which indicate a frequency difference (offset) $\Delta f$ is approximately $101 \mathrm{~Hz}$.

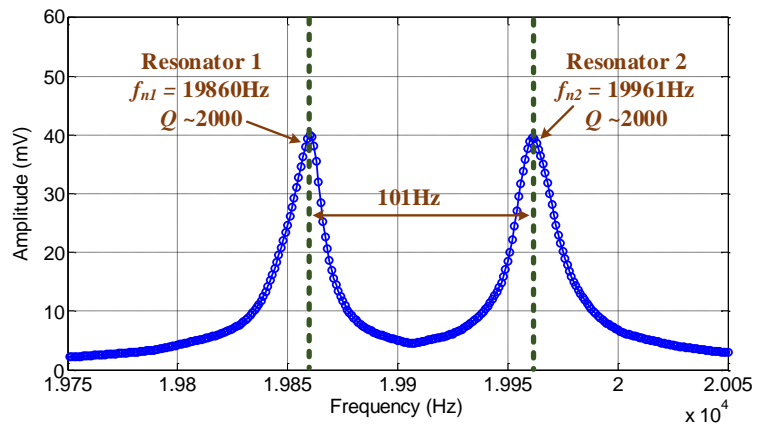

Fig.4. Frequency response of the two resonators. The frequency difference is $101 \mathrm{~Hz}$.

This dual-resonators structure is operated in drive and sense mode, respectively. The Laplace transfer function between the Lorentz force and the magnetometer output displacement can be derived as: 


$$
\left|\frac{x\left(j \omega_{d}\right)}{F_{L}\left(j \omega_{d}\right)}\right|=\frac{1}{m} \frac{1}{\sqrt{\left(\omega_{s}^{2}-\omega_{d}^{2}\right)^{2}+\left(\frac{\omega_{s}}{Q} \omega_{d}\right)^{2}}}
$$

In case the frequency difference (offset) $\Delta f$ is much lower than the resonant frequency $(\sim 20 \mathrm{kHz})$ and is also much larger than the mechanical bandwidth, this transfer function can be reasonably approximated as almost flat around the operating point. Equation (11) can be simplified to:

$$
\begin{aligned}
& \left|\frac{x\left(j \omega_{d}\right)}{F_{L}\left(j \omega_{d}\right)}\right| \approx \frac{1}{m} \frac{1}{\omega_{s}^{2}-\left(\omega_{s}-\Delta \omega\right)^{2}} \approx \frac{1}{m} \frac{1}{2 \omega_{s} \Delta \omega-\Delta \omega^{2}} \\
& \approx \frac{1}{k} \frac{\omega_{s}}{2 \Delta \omega}=\frac{1}{k} Q_{\text {eff }}
\end{aligned}
$$

Where $\left(\omega_{d} \omega_{s} / Q\right)^{2}$ and $\Delta \omega^{2}$ were neglected in this calculation process, respectively; $Q_{\text {eff }}$ represents an effective quality factor of the design magnetometer. The bandwidth cannot be extended without loss in resolution when operating at resonance. On the contrary, when operating in a frequency difference mode $(\Delta f)$, the $\pm 3 \mathrm{~dB}$ bandwidth is extended to $>\Delta f / 3$, which is independent from the damping coefficient. However, off-resonance mode achieved maximum bandwidth in sacrificing signal-to-noise ratio and resolution.

\section{SYSTEM MODELING AND SIMULATION}

\section{A. System-level simulations}

The schematic view of the dual-resonator magnetometer and corresponding system-level Simulink model is shown in Fig.5(a) and Fig.5(b), respectively. The capacitance signal readout is done by a trans-impedance amplifier for front-end capacitance to voltage $(C / V)$ conversion. Then, the signal from the $C / V$ circuit is transferred to the digital part for further signal processing, thus a multi-bit quantizer is used for $\mathrm{A} / \mathrm{D}$ conversion. The drive loop consists of the first resonator embedded in an electrostatic force closed-loop forming a self-oscillator. This self-oscillation loop uses an embedded digital phased locked loop (PLL) to achieve constant amplitude at first frequency $\left(f_{n l}\right)$. Additionally, this self-oscillation signal is converted to a clean reference clock, generating the whole system clock (CK) and the sampling frequency $\left(f_{s}\right)$. Hence, the control system does not need an external crystal, which leads to a lower clock noise floor. Moreover, this self-clocking scheme provides a fixed ratio between $f_{s}$ and the second resonator resonance frequency $\left(f_{n 2}\right)$ which also acts as the Lorentz force signal carrier. Once the drive oscillation loop is stable, a bias current driver consisting of an improved Howland current pump is used to provide a driving current $I_{\mathrm{ac}}$ with a frequency of $f_{n l}$. When an magnetic field $(B)$ is applied, driving current $I_{\text {ac }}$ produces a Lorentz force $\left(F_{L}\right)$ which induces displacement motion in the sense mode resonator that is measured by using parallel-plate capacitive fully differential electrodes $S+$ and $S$ The front-end $C / V$, boost amplifier and force feedback gain can be modeled at system level as simple gain constants $K_{p o}, K_{b s t}$ and $K_{f b}$, respectively. The sense mode (second) resonator is embedded in a sigma-delta modulator that forms an high-order
$\mathrm{EM}-\Sigma \Delta \mathrm{M}$ force rebalance control loop. The further processing steps include demodulation and decimation filtering which are also employed to produce the final output voltage signal which is a measure of the magnetic field strength $(B)$.

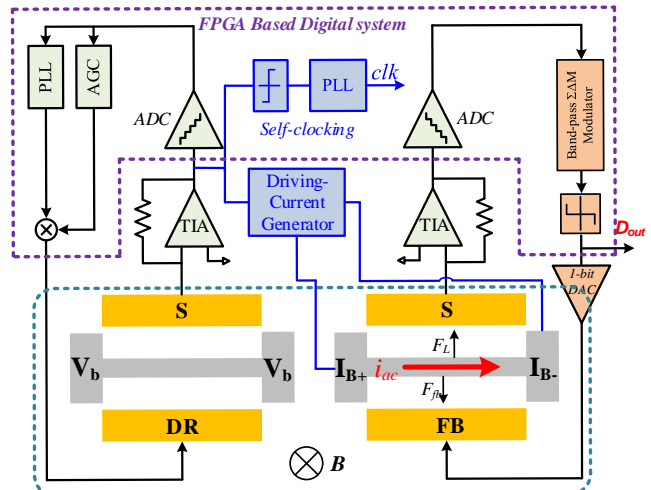

(a)

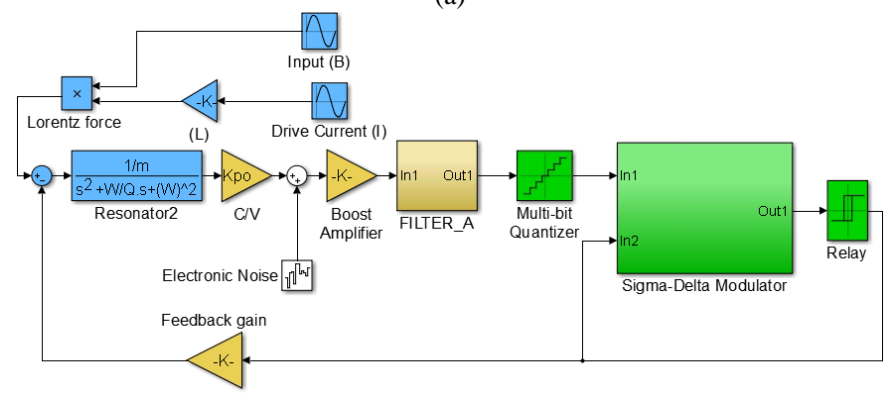

(b)

Fig.5. (a) Schematic view of the magnetometer consist of self-oscillation loop and EM- $\Sigma \Delta \mathrm{M}$ sensing loop. (b) System-level EM- $\Sigma \Delta \mathrm{M}$ sensing model

The simulated power spectrum density (PSD) of the dual quantization EM- $\Sigma \Delta \mathrm{M}$ Lorentz force magnetometer system is shown in Fig.6(a); the noise floor is about $-150 \mathrm{~dB}$, the SNR is larger than $100 \mathrm{~dB}$ in a bandwidth of $64 \mathrm{~Hz}$ with a $10 \mathrm{mT}, 32 \mathrm{~Hz}$ magnetic field input. As it is shown, the modulator noise shaping notch is centered at $19.961 \mathrm{kHz}$ (the resonant frequency of the second resonator). As shown in Fig.6(b), the Lorentz force magnetometer displacement in closed-loop operation was reduced significantly compared to open-loop operation.

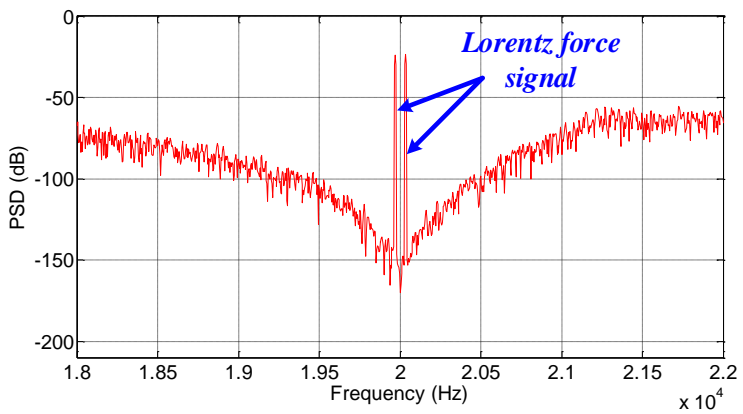

(a) 


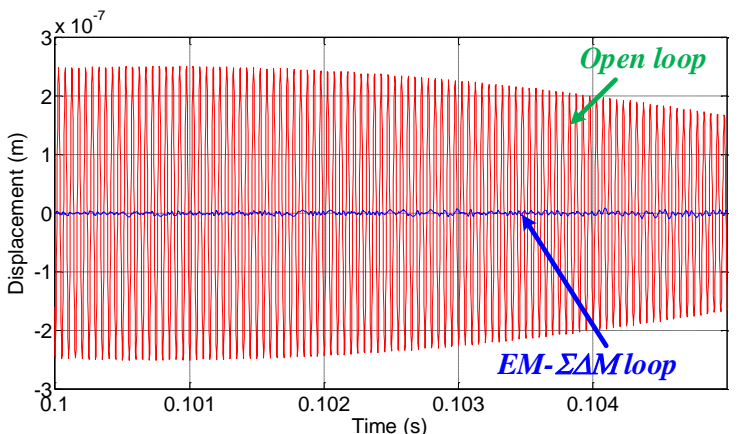

(b)

Fig.6. (a) Simulated output power spectrum density (PSD) of the EM- $\Sigma \Delta \mathrm{M}$ magnetometer (b) Comparison between the Lorentz force displacement in open-loop and closed-loop operation with $10 \mathrm{mT}, 32 \mathrm{~Hz}$ magnetic field input.

\section{B. Current generating circuit}

The current generating circuit is designed to convert the first resonator self-oscillation voltage signal into a driving current signal $\left(i_{a c}\right)$. Fig.7(a) shows the schematic of an improved Howland current pump, which is an operational amplifier topology forming a linear voltage to current convertor and also providing high output resistance. The generated driving current is pumped into the resonant structure and avoids unwanted electrostatic forces, which can result in an output signal error. An ancillary circuit is used to sample the voltage at this end of spring using a high-impedance buffer and an 180deg phase shifted voltage is applied at the other end of the springs through an inverting buffer. Fig.7(b) show signals at the end of the springs with balanced driving. The middle point of the springs is kept at a constant voltage, and the two ends basically have an anti-phase voltage behavior. There might be a residual signal because of the springs' resistance mismatching error and circuit non-idealities. However, this is negligible and does not generate a measurable output signal.

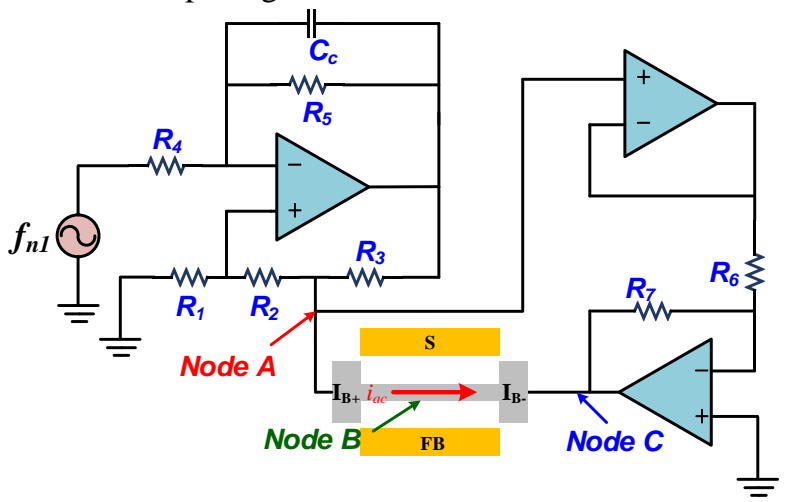

(a)

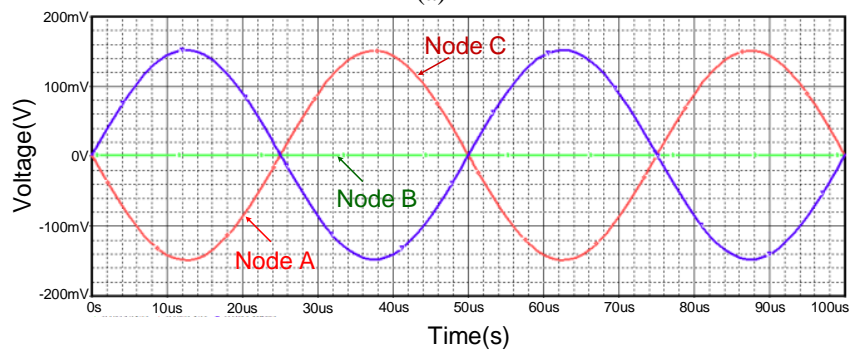

(b)

Fig.7. (a) Schematic of an improved Howland current pump with minimized residual signal at vibratory structure. (b) Balanced scheme for magnetometers current excitation with a relevant reduction of unwanted electrostatic forces.

\section{EXPERIMENTAL SETUP AND RESULTS}

As shown in the block diagram in Fig.8, the electronic circuit system is divided into analog and digital circuit blocks. The $\mathrm{EM}-\Sigma \Delta \mathrm{M}$ is fitted with capacitive readout components that are designed with a fully differential path to eliminate common mode electronic noise signals within the system. The output signal of the capacitive readout is then converted into a digital signal using a 18-bit ADC. In order to force rebalance, the capacitive actuators $(F B)$ are excited by the feedback pulse signals. The digital circuit part was implemented using a Xilinx FPGA chip. The FPGA unit reads the 18-bit data performs the $\Sigma \Delta \mathrm{M}$ filtering, and then outputs the feedback switching signals and magnetic field output. The system is designed to operate with RS-422 serial port to transmit real-time data.

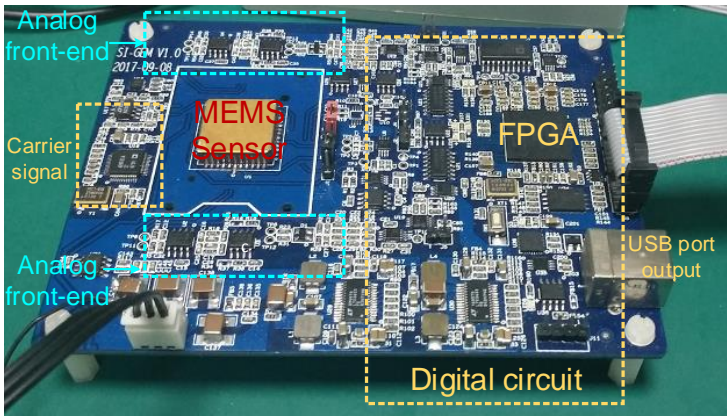

Fig.8. Electronic circuit showing the different parts of the system with the Lorentz force magnetometer sensor

Due to high- $Q$ amplification, this magnetometer operating with mode-matching offer higher sensitivity and resolution. The $f_{n 2}$ is measured higher than the $f_{n 1}$ which allow mode matching despite process variations. Mode matching $\left(f_{n 2}=f_{n 1}\right)$ is achieved by increasing the voltage difference between the sigma delta feedback pulses $\left(V_{F H}+V_{F L}\right) / 2$ and DC polarization voltage $\left(V_{P}\right)$ of second structure in Fig.9(a). The experimental setup is based on a three-axial Helmholtz coil and the closed-loop magnetic field compensator. This instrument is also equipped with an reference magnetometer and is capable of precisely measuring magnetic field enclosed by the coils and compensating it. As shown in Fig.9(b), The packaged Lorentz force magnetometer was assembled with the interface electronic circuit, and placed at the center of the Helmholtz coil to avoid effects due to field non-uniformity near the coils.

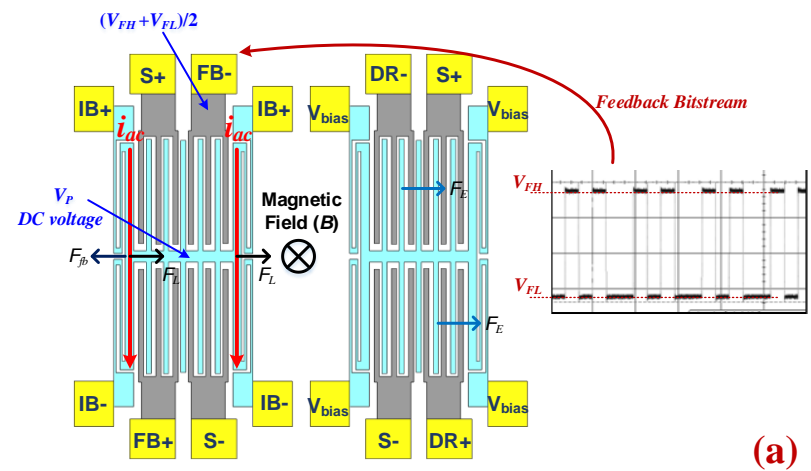




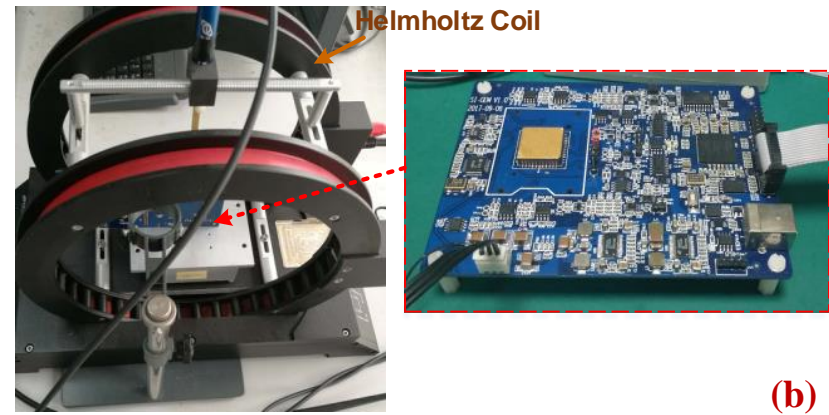

Fig.9. (a) Schematic of the proposed electrostatic mode-matching mechanism (b) Photograph of the MEMS magnetometer experimental setup that sensor was tested using a Helmholtz coil.

The first resonator drive circuit needs to provide robustness to parasitic mechanical resonant modes, and also provide a low phase-noise clock for the entire system. The drive architecture of this work embeds the PLL inside the drive loop, which also produces a high frequency clock for the system. The PLL of this work uses a $19.86 \mathrm{kHz}$ digital sinusoidal input from ADC connected to the drive detection capacitors. It then uses a phase detector (PD) to demodulate this signal with a reference sinusoid of $19.86 \mathrm{kHz}$ generated digitally from a look-up table, clocked synchronously with the NCO (numerically-controlled -oscillator) and synchronized with the phase of the drive signal. This clean reference clock for an external PLL, which generates the $30 \mathrm{MHz}$ system clock and the $625 \mathrm{kHz}$ sampling frequency. Fig.10 shows the measured phase noise density-to-carrier versus carrier offset frequency for the self-clocking clock, which is as low as $-110 \mathrm{dBc} / \mathrm{Hz} @ 1 \mathrm{kHz}$ for the system.

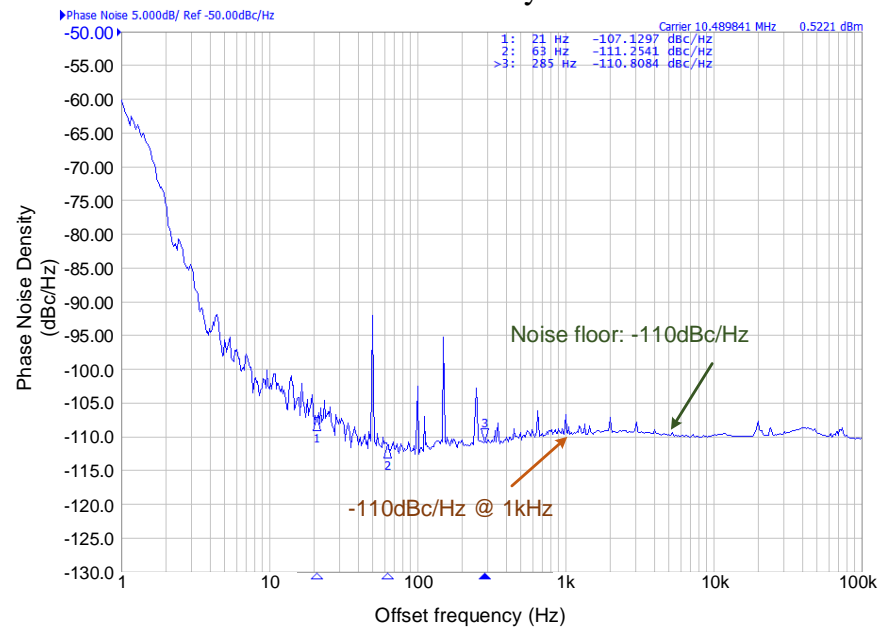

Fig. 10. Measured phase noise performance of dual-resonator self-clocking

For each measurement, the magnetometer is driven at its resonance frequency with a current of $1 \mathrm{~mA}$ which is delivered by the Howland pump. The output bitstream is decimation and demodulated at the natural frequency $f_{n l}$, and passed through a low-pass filter to filter out the harmonics. The Helmholtz coil first compensates the environmental magnetic field and then generates steps of increasing field values for the Z-axis from $0 \mu \mathrm{T}$ to $400 \mu \mathrm{T}$, in $0.5 \mu \mathrm{T}$ increments (keeping nominally null the magnetic field over the other directions). As shown in Fig. 11(a), the measured open-loop and closed-loop DC sensitivity was $21.2 \mu \mathrm{V} / \mu \mathrm{T}$ and $13.2 \mu \mathrm{V} / \mu \mathrm{T}$, respectively. The system maximum dynamic range is limited by the generated feedback voltage in this circuit. Fig. 11(b) shows the measured noise spectra of the final output. The $1 / \mathrm{f}$ noise corner frequency is about $60 \mathrm{mHz}$, reaching the magnetometer noise floor limit of at $1 \mu \mathrm{T} / \sqrt{ } \mathrm{Hz}$ at higher frequencies.

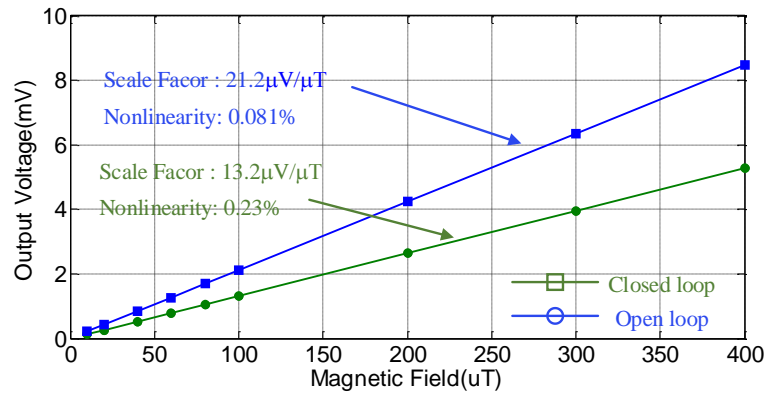

(a)

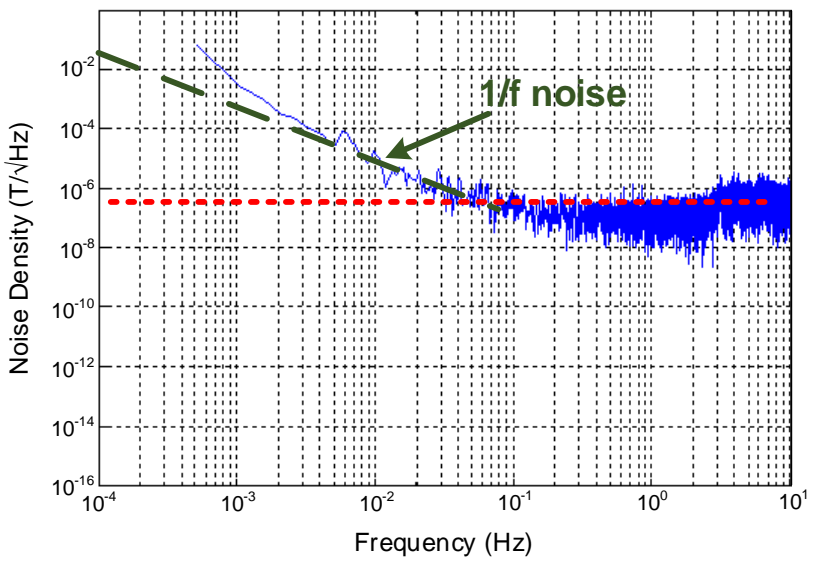

(b)

Fig.11. (a) output voltage as a function of the input magnetic filed and (b) measured noise spectra of the implemented magnetometer output.

Fig.12 shows the frequency response of the presented self-clocked EM- $\Sigma \Delta \mathrm{M}$ magnetometer, obtained by sweeping the frequency of a constant amplitude AC magnetic field and measuring the corresponding output amplitude. The bandwidth $(B W)$ of the Lorentz force magnetometer can be extended to $\sim 80 \mathrm{~Hz}$ in $\mathrm{EM}-\Sigma \Delta \mathrm{M}$ closed-loop operation compared to AM open-loop operation, in which the bandwidth is only $5 \mathrm{~Hz}$. It was thus experimentally shown that the bandwidth is improved by $16 \mathrm{X}$ in the EM- $\Sigma \Delta \mathrm{M}$ operation compared to the AM open-loop operation, independently from the mechanical bandwidth $\left(B W_{\text {mech }}\right)$.

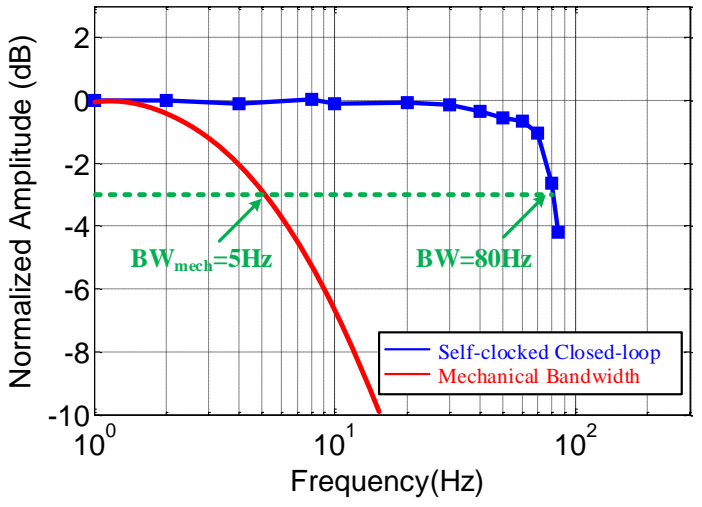

Fig.12. Frequency response of the self-clocking EM- $\Sigma \Delta \mathrm{M}$ magnetometer achieved 16X greater bandwidth than the mechanical bandwidth.

The Lorentz force magnetometer's startup bias output was 
recorded immediately while the interface circuit system was powering on. The device was tested in a magnetically shielded environment without any temperature control. Fig.13(a) shows the measured output bias drift of the open-loop and self-clocked EM- $\Sigma \Delta \mathrm{M}$ closed-loop, respectively. The proposed self-clocked closed-loop method significantly reduces the effect of $T C F$, and improving the zero-field output bias instability. Allan deviation is used to measure the bias instability of the magnetometer in open-loop and self-clocked closed-loop, shown in Fig.13(b). The measured bias instabilities of the open-loop and self-clocked closed-loop are $1 \mu \mathrm{T} @ 1 \mathrm{~s}$ and $0.6 \mu \mathrm{T} @ 8 \mathrm{~s}$, respectively. At $8 \mathrm{~s}$ averaging time, the self-clocked EM- $\Sigma \Delta \mathrm{M}$ magnetometer exhibits 7-fold better performance than the open-loop device.

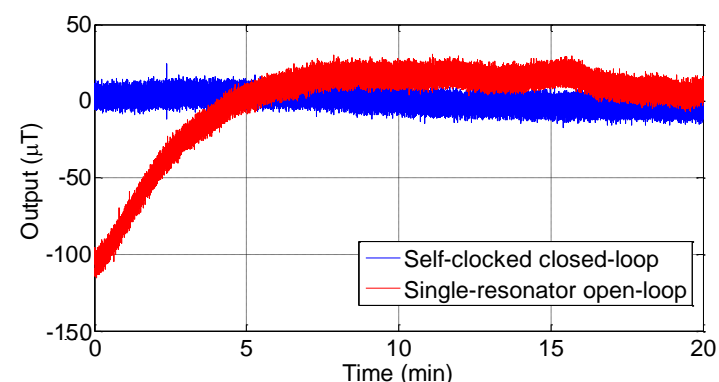

(a)

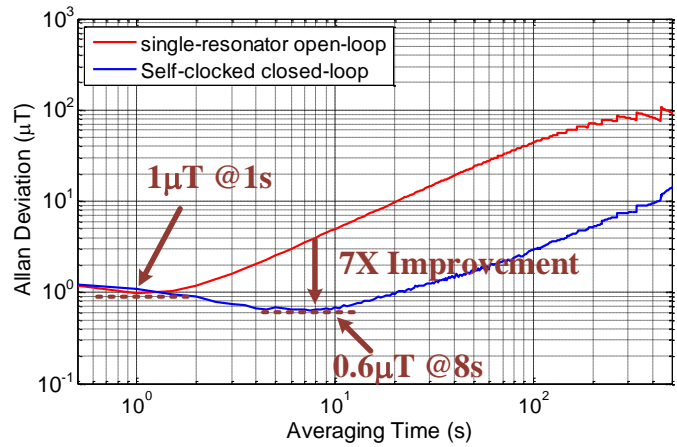

(b)

Fig.13. (a) Measured bias output of magnetometer in self-clocked closed-loop and open-loop mode during power on. (b) Allan deviation of the measured zero-field output data.

Typical temperature sensitivity of the resonant frequency for silicon MEMS structure is $-30 \mathrm{ppm} /{ }^{\circ} \mathrm{C}$ at near room temperature. A major drift source for Lorentz force magnetometer is still their inherent sensitivity to temperature variations. Drift output can be modeled as a constant plus a time-varying component, which is caused by the temperature fluctuations. Conventional approaches for Lorentz force magnetometer calibration rely on external temperature sensors, which suffer from the thermal lag and temperature-induced hysteresis. Dual-resonator structure utilizes first resonator as a thermometer for the compensation of sensing mode (second resonator), which is free from any spatial or temporal thermal lag. Meanwhile, a self-clocked EM- $\Sigma \Delta \mathrm{M}$ force feedback mechanism which fixes the ration between the sampling frequency and MEMS resonance frequency. Thus, digital processing units poles and zeros scale with resonance frequency, and filters are self-tuned with the variation of the frequency. This allows for self-compensation of temperatureinduced output drifts. In fig.13(a), we demonstrate feasibility of MEMS magnetometer for turn-on bias compensation. During the turn-on event, the MEMS chip and interface electronics heats up with the temperature gradient, which in turn leads to the increase in bias drift. Self-clocked resonance frequency change is indicative of the magnetometer temperature change, and removed the drift trend and enabled an improvement since the cold-start, proving feasibility of the approach.

\section{CONCLUSION}

This paper has described a novel dual-resonator EM- $\Sigma \Delta \mathrm{M}$ Lorentz force MEMS magnetometer to improve its bias output stability. The MEMS structure has a matched pair of resonators on the same silicon die. The first is operated self-resonance in closed-loop as an oscillator to generate the driving current and system self-clocking, and the second is operated high-order $\mathrm{EM}-\Sigma \Delta \mathrm{M}$ force rebalance for magnetic field sensing. With a modest vacuum level through ceramic encapsulation, the sensor has a $Q$ of 2000 for in-plane motion. Compared to conventional open-loop operation, the operation demonstrated here enabled to operate the magnetometer in self-clocking $\mathrm{EM}-\Sigma \Delta \mathrm{M}$ force rebalance mode and with resulting in considerably bandwidth improvement and better bias instability without suffering from unreliable TCF over temperature. With an excitation current of $1 \mathrm{~mA}$, the sensor has a $\mathrm{z}$-axis field sensitivity of $13.2 \mu \mathrm{V} / \mu \mathrm{T}$ and the noise floor of $1 \mu \mathrm{T} / \sqrt{ } \mathrm{Hz}$. The MEMS sensor has a bias instability of $0.6 \mu \mathrm{T}$ with an averaging time of $8 \mathrm{~s}$. The sensor performance can also be improved by better vacuum packaging. Better vacuum will result in higher $\mathrm{Q}$ which in turn will lower the noise, while still maintain an acceptable bandwidth of $80 \mathrm{~Hz}$. The experimental measurements are proved that the EM- $\Sigma \Delta \mathrm{M}$ force-rebalance structure is applicable for high performance magnetometer.

\section{REFERENCES}

[1] S. Brenna et al.. "A low-noise sub-500 $\mu \mathrm{W}$ Lorentz force based integrated magnetic field sensing system," in Proc. $28^{\text {th }}$ IEEE Int. Conf. Micro Electro Mech. Syst. (MEMS), 2015, pp. 932-935

[2] G. Langfelder, C. Buffa, A. Frangi, A. Tocchio, E. Lasalandra, and A. Longoni, "Z-axis magnetometers for MEMS inertial measurement units using an industrial process," IEEE Trans. Ind. Electron., vol. 60, no.9, pp. 3983-3990, 2013.

[3] M. Li, V. T. Rouf, M. J. Thompson, and D. A. Horsley, "Three-axis Lorentz-force magnetic sensor for electronic compass applications," $J$. Microelectromech. Syst., vol. 60, no.9, pp. 3983-3990, 2013.

[4] M. Li, E. J. Ng, V. A. Hong, C. H. Ahn, Y. Yang, T. W. Kenny, and D. A. Horsley, "Lorentz force magnetometer using a micromechanical oscillator," Appl. Phys. Lett., vol.103, no.17, pp. 173504-1-173504-4, 2013

[5] S. Sonmezoglu, M. Li, and D. A. Horsley, "Force-rebalanced Lorentz force magnetometer based on a micromachined oscillator," Appl. Phys. Lett., vol.106, no.9, pp. 093504, 2015.

[6] G. Langfelder and A. Tocchio, "Operation of Lorentz-force MEMS magnetometers with a frequency offset between driving current and mechanical resonance," IEEE Trans. Magn., vol. 50, no.1, Art. ID 4700106, 2014.

[7] G. Langfelder, G. Laghi, P. Minotti, A. Tocchio, and A. Longoni, "Off-resonance low-pressure operation of Lorentz force MEMS magnetometers," IEEE Trans. Ind. Electron., vol. 61, no.12, pp. 7124-7130, 2014

[8] S. Sonmezoglu, D. A. Horsley, "off-resonance operation of a MEMS Lorentz force magnetometer with improved thermal stability of the scale factor," in Proc. $29^{\text {th }}$ IEEE Int. Conf. Micro Electro Mech. Syst. (MEMS), 2016, pp. 103-106 
[9] V. Kumar, S. Pourkamali, "Lorentz force MEMS magnetometer with frequency modulated output," in Proc. $29^{\text {th }}$ IEEE Int. Conf. Micro Electro Mech. Syst. (MEMS), 2016, pp. 589-592

[10] S. Sonmezoglu, I. B. Flader, Y. H. Chen, D. D. Shin, T. W. Kenny, D. A. Horsley, "dual-resonator MEMS Lorentz force magnetometer based on differential frequency modulation," in Proc. $29^{\text {th }}$ IEEE Int. Symp. Inertial Sensors Syst.(INERTIAL), 2017, pp. 814-817.

[11] M. Li et al., "Lorentz force magnetometer with quadrature frequency modulation," in Solid-State Snes., Actuators, Microsyst. Workshop Tech. Dig., Hilton Head, SC, USA, 2014, pp.52-55.

[12] M. Li, S. Sonmezoglu, and D. A. Horsley, "Extended bandwidth Lorentz force magnetometer based on quadrature frequency modulation," $J$. Microelectromech. Syst., vol. 24, no.2, pp. 333-342, 2015.

[13] F. Chen, W. Zhou, H. S. Zou, M. Kraft, X. X. Li, "Self-clocked dual-resonator micromachined Lorentz force magnetometer based on electromechanical sigma-delta modulation," in Proc. $31^{\text {st }}$ IEEE Int. Conf. Micro Electro Mech. Syst. (MEMS), 2018, pp. 940-943

[14] F. Chen, X.X. Li, and M. Kraft, "Electromechanical sigma-delta modulators $(\Sigma \Delta \mathrm{M})$ force feedback interfaces for capacitive MEMS inertial sensors: A review," IEEE Sensors J., vol.16, no.17, pp.6476-6495, 2016.

[15] B. Sheng, F. Chen, C. Qian, D. C. Xu, S. W. Guo and X. X. Li, "Design of a dual quantization electromechanical sigma-delta modulator MEMS vibratory wheel gyroscope," J. Microelectromech. Syst., vol. 27, no.2, pp. 218-230, 2018

[16] F. Chen,Y. Zhao, J. C. Wang, H. S. Zou, M. Kraft, and X. X. Li, “A single-side fabricated tri-axis (111)-silicon microaccelerometer with electromechanical sigma delta modulation," IEEE Sensors J., vol. 18 no. 5, pp. 1859-1869, 2018.

[17] F. Chen, H. L. Chang, W. Z. Yuan, R. Wilcock, and M. Kraft, "Parameter optimization for a high-order band-pass continuous-time sigma-delta modulator MEMS gyroscope using a genetic algorithm approach," $J$. Micromech. Microeng., vol. 22, no. 10, p. 105006, Oct. 2012.

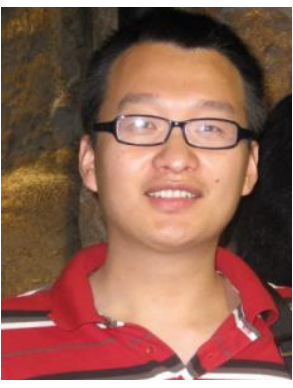

Fang Chen received the B.S., M.S., and Ph.D. degrees in mechanical and electronic engineering from Northwestern Polytechnical University (NPU), Xi'an, China, in 2006, 2009 and 2014. He was a joint training Ph.D. student with the Nano Group (Prof. Michael Kraft), School of Electronics and Computer Science, University of Southampton, UK. In 2014, he became an assistant researcher at Shanghai Institute of Microsystem and Information Technology (SIMIT), Chinese Academy of Sciences (CAS); since 2017, he is an associate professor with the SIMIT, CAS, Shanghai, China. His current research interests include MEMS inertial sensors, interface sensing and control circuits.

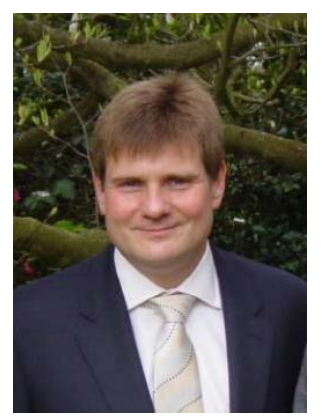

Michael Kraft received the Dipl.-Ing. (Univ.) degree in electrical and electronics engineering from the Friedrich Alexander Universität Erlangen-Nürnberg, Erlangen, Germany, in 1993 and the Ph.D. degree from Coventry University, Coventry, U.K., in 1997. He is currently a professor of Micro- and Nano-systems at the Catholic University of Leuven, Belgium, and a Guest Professor with Shanghai Institute of Microsystem and Information Technology, Chinese Academy of Sciences. Before joining the University of Leuven, from 2015 to 2017 he worked at the University of Liege, Belgium and from
2012 to 2014 he was with the Fraunhofer Institute for Microelectronic Circuits and Systems, Duisburg, Germany, heading the department of Micro- and Nano-systems with a focus on fully integrated microsensors and biohybrid systems. Concurrently, he held the professorial Chair (W3) of Integrated Micro- and Nano-systems with the University of Duisburg-Essen. From 1999 to 2012, he was an academic at the School of Electronics and Computer Science, University of Southampton, Southampton, U.K, where he also acted as the Director of the Southampton Nanofabrication Centre. He also spent two years at the Berkeley Sensors and Actuator Centre, University of California, Berkeley, working on integrated MEMS gyroscopes. In his career he has focused on novel micro- and nanofabrication techniques, microsensors, and actuators and their interface circuits, particularly for capacitive sensors. He has a broad interest in MEMS and nanotechnology ranging from process development to system integration of MEMS and nanodevices. He has published over 250 peer-reviewed journal and conference papers as an author or coauthor. He also contributed to three text books on MEMS and edited a book on MEMS for aerospace and automotive applications.

Dr. Kraft has served on several steering and technical committees of international conferences such as IEEE Sensors, Eurosensors, ISSCC and the Micromechanics and Microsystems Europe Workshop.

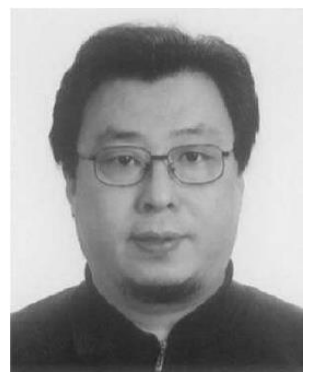

Xinxin Li received the B.S. degree in Semiconductor physics and devices from Tsinghua University, Beijing, China, in 1987, and the Ph.D. degree in microelectronics from Fudan University, Shanghai, China, in 1998. For a long period time, his research interests have been in the fields of micro/nano sensors and MEMS/NEMS. $\mathrm{He}$ was a research engineer with Shenyang Institute of Instrumentation Technology, Shenyang, China, for five years. He was also with Hong Kong University of Science and Technology, Kowloon, Hong Kong, as a Research Associate and with Nanyang Technological University, Singapore, as a Research Fellow. He then joined Tohoku University, Sendai, Japan, as a Lecturer (Center of Excellence Research Fellowship). Since 2001, he has been a professor with the Shanghai Institute of Microsystem and Information Technology, Chinese Academy of Sciences, Shanghai. From 2007 he has been serving as the Director of the State Key Laboratory of Transducer Technology, China. He has invented more than 80 patents and published more than 300 papers in refereed journals and conference proceedings (including about 160 SCI papers). He is on the Editorial Board of the Journal of Micromechanics and Microengineering. Prof. Xinxin Li served as the technical Program Committee member for IEEE MEMS conference 2008, 2011 and 2017, IEEE Sensors conference from 2002 to 2014 . From 2014, he has been serving as an International Steering Committee member for the conference on Solid-State Sensors, Actuators and Microsystems (Transducers). 